schildert Barley in seinem Buch «Die Raupenplage` in fast komödiantischer Weise, wie er sich auf den Weg zu einem afrikanischen „Stamm“ macht, weil er über ethnologische Buschtrommeln gehört hat, daß dort ganz spezifische Initiationsriten bestünden, bei denen die Brustwarzen von männlichen wie weiblichen Jugendlichen vor dem Eintritt ins Erwachsenenalter beschnitten würden. Das wäre eine ethnologische Sensation, und Barley ist ihr auch auf der Spur, als er nach einigen Verwirrungen endlich auf jenen "Stamm“ trifft, bei dem er tatsächlich Männer und Frauen ohne Brustwarzen sieht. Nun geht es nur noch um die endgültige Dokumentation der Beschneidungszeremonien, und ein neues ethnologisches Sensationsbuch wäre geboren. Doch lassen diese Zeremonien lange auf sich warten. Ungeduldig traut Barley sich schließlich, genauer nachzufragen, als er eine solche ,initialisierte“ Frau sieht. Das Ergebnis ist verblüffend: „Alle lachten. Natürlich war sie so zur Welt gekommen. Wer würde sich schon die Brustwarzen abschneiden? So etwas war doch schmerzhaft. Nun war also klar, daß die Ninga, was immer sonst noch mit ihnen passiert sein mochte, jedenfalls Opfer genetischer Mißbildungen waren. Der Klumpfuß und der Zwergwuchs des Häuptlings, der Buckel seines Bruders, die mißgebildeten Brustwarzen bei allen waren insgesamt Folgen ein und derselben angeborenen Abnormität und nicht, wie ich geglaubt hatte, Ausdruck kultureller Symbolik." (Barley I986: I04)

\title{
2. Mikrohistorie: Quellenerschließung und Quellenkritik
}

$\mathrm{Da}$ in dieser Übersicht nach der Feldforschung, die sich primär gegenwartsbezogenen Themen zuwendet, gleich die Mikrohistorie folgt, hat mehrere Gründe. Zum einen soll es die Forderung unterstreichen, daß sich Europäische Ethnologie intensiv auch mit der geschichtlichen Dimension der Kultur zu befassen hat. Denn Gegenwart wird nur verständlich in ihrer geschichtlichen Entwicklung, und Geschichte wird uns in Bildern und Begriffen verfügbar nur durch Fragestellungen aus der Gegenwart - so sehr manche Historiker eine unabhängige „Wirklichkeit“ der Vergangenheit beschwören mögen. Zum zweiten wird historische Forschung in vielen Bereichen längst unter intensiver Einbeziehung ethnologischer und kulturanthropologischer Perspektiven betrieben. Dabei spielt insbesondere die Erkenntnis eine Rolle, daß auch das historische „Feld“ eine andere Kultur verkörpert, mit der wir in Austausch treten und die damit umgekehrt auch unsere Leseweisen problematisiert und reflektiert: „Daß Untersuchungsstrategien längst nicht immer so aufgehen, wie man sich das ursprünglich ausgemalt hat, daß die Erfahrungen 
im Forschungs-,Feld' eine fortwährende, die eigene Arbeit kontinuierlich begleitende Irritation darstellen, daß diese Irritationen, aber auch die Irrwege und Sackgassen, auf die man auf schlecht ausgeschilderten Forschungswegen und Sackgassen immer wieder gerät, in der Darstellung nicht, glattgebügelt ${ }^{\star}$ werden müssen, sondern selbst ein tragendes Element der Erkenntnis darstellen dürfen, macht vielleicht die eigentliche Stärke der ethnohistorischen Betrachtungsweise aus." (Schindler I992: I8) Noch ein drittes verbindet die Feldforschung mit der Mikrohistorie: Beide sind charakterisiert durch die konzentrierte Beoachtung eines kleinen, überschaubaren Untersuchungsfeldes, in dem räumliche, zeitliche und soziale „Nahaufnahmen" mit hoher Tiefenschärfe erzielt werden sollen. Für die Geschichte liegen die Gründe dafür ebenso auf der Hand wie für die Gegenwart: Kultur wird am allerwenigsten in statistischer Repräsentativität faßbar, sondern nur über Einblicke in exemplarische und charakteristische Situationen, über Einsichten in das Zusammenspiel verschiedenartiger Faktoren und über die Beobachtung konkreter Praxen von identifizierbaren Individuen und Gruppen. Doch ist es ein räumliches und kein semantisches „Klein“: Es meint nicht „Kleinigkeiten", sondern den Ausschnitt eines großen Feldes samt den darin wirkenden gesellschaftlichen Prozessen und Problemlinien. Dieser Ausschnitt ist in seinem Verhältnis zum Ganzen systematisch zu bestimmen und methodisch wie eine fotografische Ausschnittvergrößerung zu behandeln, die mehr Details freigeben kann als der gesamte Prospekt, jedoch immer vor dessen Hintergrund betrachtet werden muß.

Mikrohistorie verkörpert insofern meist auch einen erfahrungsund alltagsgeschichtlichen Zugang, betont nur begrifflich stärker dessen methodische Seite. Dabei beabsichtigt sie nicht primär eine „Geschichte der kleinen Dinge“ und der unpolitischen Nischen, sondern einen Einblick in die Mikrophysik historischer Gesellschaft im Sinne von deren lebensweltlichen Funktionsweisen und kulturellen Regelsystemen (Lüdtke 1994). Auch darin ist sie wiederum aufs engste dem Verständnis ethnologischer Feldforschung verbunden, die Bedingungen, Regeln und Formen gegenwärtig gelebter Erfahrung untersuchen will. Diese interdisziplinäre Perspektive signalisieren im übrigen auch die inzwischen etablierten Richtungsangaben Historische Anthropologie oder Ethnohistorie.

\section{„Der Käse und die Würmer"}

Dennoch ist der Begriff Mikrohistorie keine volkskundliche oder ethnologische Prägung, sondern er stammt aus den Historikerdebatten der I970er Jahre. Und er ist auch weder ein epistemologisch ver- 
bindlicher noch ein theoretischer oder systematischer Begriff, eher der Kennzeichnungsversuch einer bestimmten Geschichtsperspektive und eines bestimmten Umgangs mit den Methoden der Quellenforschung. Mir scheint er als Leitbegriff für solche spezifisch kulturwissenschaftlich orientierte Zugangsweisen zur Geschichte durchaus nützlich zu sein, wenn man sich darüber einig ist, daß darunter auch wesentliche Teile einer Alltagsgeschichte oder einer Geschlechtergeschichte zu subsumieren sind.

Der italienische Kulturhistoriker Carlo Ginzburg, einer der Mitbegründer und wichtigsten Vertreter einer Mikrohistorie, erinnert sich in einem Artikel an seine erste Bekanntschaft mit diesem Begriff: „Ich glaube, es war Giovanni Levi, den ich I977 oder I978 zum ersten Male von ,Mikro-Historie' habe sprechen hören. Vermutlich übernahm ich dieses Wort - dem ich niemals zuvor begegnet war -, ohne nach seiner eigentlichen Bedeutung zu fragen: Ich werde mich ganz einfach mit dem Bezug auf ein reduziertes Beobachtungsfeld zufrieden gegeben haben, wie es das Präfix ,mikro“ nahelegt." Ginzburg weist dann darauf hin, daß der amerikanische Historiker George R. Stewart allerdings schon in den I95oer Jahren diesen Begriff für seine eigene Geschichtsschreibung verwandt hatte (Ginzburg I993b: I69). Andere wie der bekannte französische Historiker Fernand Braudel hatten diesen Begriff ebenfalls bereits zuvor benutzt, ohne daß sich aus diesen Ansätzen jedoch ein weiterführendes Konzept ergeben hatte.

I976 veröffentlicht Carlo Ginzburg eine bald vielbeachtete mikrohistorische Studie unter dem Titel (Der Käse und die Würmer. Die Welt eines Müllers um i600). Darin wird der Prozeß eines Müllers aus dem italienischen Friaul dargestellt, der von der römischen Inquisition der Gotteslästerung angeklagt ist, weil er ganz eigene Ansichten über die Entstehung der Welt, über Gott und über viele Glaubensfragen vertritt. Die Verhöre und der Prozeß sind in vielseitigen Gerichtsprotokollen dokumentiert, in denen detailliert festgehalten ist, mit wem der Müller in seinem Dorf und seiner Region sozialen Umgang hatte, wie er an Bücher kam und welche er las, wie er seinen religiösen Pflichten nachging und wie er sich die Entstehung der Welt erklärte, seine „Kosmogonie“. Aus den aufgeführten Informations- und Bildungsquellen setzte sich dieser Müller ein eigenes Bild der Schöpfungsgeschichte zusammen, das ihm die Entstehung der Welt wie einen chemischen Gärungsprozeß erscheinen ließ, wie in jenem Bild vom „Käse und den Würmern“. Ohne hier weiter auf die Geschichte selbst einzugehen, sei nur der historischen Ordnung halber festgestellt, daß dieser Müller sich als zu intelligent für die Inquisition erwies, weshalb diese ihn als „Ketzer“ schließlich zum Tode verurteilte (Ginzburg I983a). 
Nun ist bereits kulturgeschichtlich erstaunlich, daß uns die Quellen das Bild eines Mannes zeichnen, der als Angehöriger des angeblich ,illiteraten Volkes“ um I60o intensiv religiöse Schriften wie philosophische und naturwissenschaftliche Werke liest, über deren Inhalt er mit Pfarrern und Adligen wie mit Dorfbewohnern spricht. Sicherlich ist dieser Müller mit seiner Lesepraxis damals eine Ausnahme, aber diese Ausnahme bestätigt doch, daß auch die ,kleinen Leute" in der Geschichte sich keineswegs nur ihren „kleinen Alltagen“ widmeten, sondern auch in darüber hinausreichenden Horizonten dachten (Chartier 1990). Gerade die Geschichte der sogenannten Ketzerbewegungen der Frühen Neuzeit wird, wenn sie erst intensiver erschlossen ist, sicherlich noch weitere Überraschungen dieser Art eröffnen.

Noch erstaunlicher ist freilich, was Ginzburg bei seinen Archivstudien aus diesen Quellen herausgelesen hat. Denn bei ihm gewinnt diese Figur des Müllers eine Plastizität und Individualität, die man aufgrund der mentalitätsgeschichtlichen Vorstellungen wie der Quellenlage bis dahin nur den Vertretern der gebildeten Schichten zugestanden bzw. zugeschrieben hatte. Lebensgeschichten wie die des Müllers erschienen als ein nicht zugängliches historisches Terrain, als eine terra incognita, die im übrigen auch nicht untersuchenswert sei, weil man die Umrisse dieses ,dumpfen Lebens in der unaufgeklärten Masse" zu kennen glaubte. Nun gewinnt ein Gesicht aus dieser anonymen Masse plötzlich ausgeprägte Züge einer Persönlichkeit. Eine Lebenswelt und Kultur werden sichtbar, die zeigen, wie wenig diese arroganten Bilder, die über eine lange kulturgeschichtliche Tradition verfügen, bei genauerem Hinsehen und bei genauerem Studium der Quellen zutreffen. Hier zeigt sich wiederum, daß ein verändertes Erkenntnisinteresse, welches sich in den I970er Jahren neu auf die „Geschichte der Vielen“ konzentriert, auch neue Erkenntnisse zutage fördern kann. Es sind nicht nur die Aktenfunde, sondern mehr noch die weiter geöffneten und anders lesenden Augen des Historikers, die hier Neues sichtbar machen.

\section{Bilder vom Anderen in der Geschichte}

So wird die Geschichte dieses Müllers zu einer ersten lebensweltlichen Studie in jenem Bereich einer vormodernen und weithin illiteraten Volkskultur, in der die frühere Volkskunde nur kollektive Lebensformen und traditionale Mythen wirken sah. Und sie wird zum Vorbild für ähnliche Studien, die seit Mitte der I970er Jahre in Italien, Frankreich, England, den USA und in Deutschland dazu beitragen, daß das Bild eines „,dunklen“ Mittelalters und einer ,verhangenen“ Neuzeit erhellt und neu geschrieben wird. ${ }^{63}$ Damit sind Ein- 
blicke in die geschichtliche Landschaft eröffnet, die den neuen Fragen einer Erfahrungs- und Alltagsgeschichte, einer Geschlechtergeschichte und einer Geschichte des Individuums, insgesamt also einer Geschichte kultureller Praxen und mentaler Dispositionen, neue Horizonte eröffnet haben. Dies wurde möglich, weil die Mikrohistorie neue „Subjekte“ der Geschichte und deren quellenmäßige Spuren in den Mittelpunkt der Betrachtung rückte (W. Schulze I988).

Ginzburgs Studie macht diese doppelte und in sich verschränkte Wirkung eines veränderten historischen Blickes und eines anderen Lesens historischer Quellen dabei ebenso exemplarisch deutlich, wie sie auch die methodischen und methodologischen Probleme dieser historischen Forschung thematisiert. Im Falle jenes Müllers gelingt die Rekonstruktion seiner Denk- und Vorstellungswelten allein auf der Grundlage der Akten der Inquisition. Keine Zeile des Müllers ist von dessen eigener Hand überliefert, sondern alles von seinen Gegnern notiert: von den Gebildeten, den Richtern, den Herrschenden, denen alle Gedanken des Müllers als „subversiv“ erscheinen, die sein ganzes Denken und seine Logik nicht verstehen, weil sie nicht verstehen wollen. Dieses Quellen- und Leseproblem läßt sich verallgemeinern: Je weiter wir in die Geschichte zurückgehen, desto mehr repräsentieren die Quellen Dokumente der Tätigkeit von Verwaltungen, Bildungseliten und Kirchenpersonal. Die Menschen außerhalb dieser Institutionen und ihr Verhalten, also die „Kultur des einfachen Volkes“, werden überwiegend durch fremde Hand beschrieben und mit fremden Augen betrachtet. Damit ergibt sich eine typisch ethnologische Problemstellung: Wie wird dieses Bild vom Anderen gezeichnet, wie das Selbstverständnis der so Beschriebenen dargestellt, mit welchem Selbstverständnis notiert der Schreiber? Wie läßt sich umgekehrt dieses Andere möglichst in dessen eigenen Begriffen fassen, aus dessen verbliebenen Bildern herauslesen?

Im Falle des Müllers verweist dieses Problem der fremden Handschrift und ihres Lesens auf ganz unterschiedliche Voraussetzungen: die Fähigkeit, historische Handschriften zu entziffern; die Kenntnis zeitgenössischen Sprachgebrauchs, wenn es um gewisse Begriffe und Bilder geht, deren spezifische historische Semantik sich nur über den Vergleich mit anderen Dokumenten etwa zeitgenössischer Literatur, kirchlicher oder politischer Verwaltungssprache erschließen läßt; das Wissen um ideen- und geistesgeschichtliche Hintergründe der Zeit, die über die jeweilige Definition von Glaubens- wie Aberglaubensformen Aufschluß geben können; die Kenntnis des sozial- und politikgeschichtlichen Hintergrunds, der verständlich macht, weshalb der „richtige Glaube“ damals so wichtig war und was er für die Aufrechterhaltung politisch-gesellschaftlicher Herrschaft bedeutete; das Verständnis des kulturgeschichtlichen Hintergrunds, um nachvollzie- 
hen zu können, wie sich Bildungselite und Volk gegenseitig sahen und was sie voneinander wußten. Jedes Quellenstück verkörpert insofern einen historisch verfertigten komplexen Text mit dichten Informationen, die mehr enthalten als ihr reiner Wortlaut, deren Bedeutung sich nur über die Kenntnis seiner sozialen Verfertigungskontexte erschließen läßt. Dies stellt hohe Anforderungen an eine Quellenkritik, die uns der Validität, der Zuverlässigkeit der Quellen versichern soll. Und „Quellen“ in einem umfassenden Sinn sind das einzige, was uns die Geschichte von sich überlassen hat: Zeugnisse früherer Menschengenerationen, die „unbewußte“, d.h. ungeplante Überlieferungen verkörpern wie Möbel, Bräuche oder Arbeitsgeräte oder die „,bewußt“ erhalten geblieben sind wie etwa Texte, Kunstgegenstände oder Denkmäler, die also absichtsvolle Linien der Tradierung verkörpern.

\section{Geschichte in "dichter Beschreibung"}

Ein vorzügliches Beispiel für solch eine systematische mikrogeschichtliche Quellenerschließung, die auf den Spuren Ginzburgs wiederum neue Horizonte einer Körper- und Geschlechtergeschichte eröffnet, bildet die Fallstudie der Tübinger Kulturwissenschaftlerin Sabine Kienitz über Sexualität und Geschlechterbeziehungen zu Anfang des I9. Jahrhunderts. Sie untersucht am Beispiel eines Prozesses in der württembergischen Kleinstadt Schwäbisch Hall, wie der wohl innerste Bezirk menschlicher Sozialbeziehungen, die Welt des Sexuellen und die Ordnung der Geschlechter, beschaffen sind bzw. wie sie sich uns über ihre quellenmäßige Erschließung verständlich machen können. In diesen Kriminalprozeß waren damals rund I 50 Personen verwickelt, einerseits Frauen und junge Mädchen aus den unteren sozialen Schichten, die der Prostitution, des Betrugs und der organisierten Erpressung angeklagt wurden, andererseits städtische Honoratioren, die sexuelle Delinquenten und Erpressungsopfer zugleich waren. Wohl aus materieller Not hatten sich die Frauen auf sexuelle Beziehungen eingelassen, um anschließend - meist unter dem Vorwand einer eingetretenen Schwangerschaft - mehr finanzielle Entschädigung zu fordern, als die Männer bereit waren zu geben. Dabei handelt es sich vor allem wiederum um Gerichtsakten, aus denen die sozialen Akteure ,sprechen“ und aus denen die Sinngebungen und Deutungen, die in den Aussagen enthalten sind, rekonstruiert werden müssen.

Es handelt sich um eine historische Fallstudie über ein lokales Ereignis. Jedoch werden lokale Welt und große Welt hier so geschickt verbunden, daß nicht etwa das Bild einer "geschichtlichen Insel“" entsteht, sondern eine differenzierte Ansicht einer Geschichtsland- 
schaft, ihrer sozialen Lebenswelten und ihrer Akteure. Sorgfältig werden die Eigen- und Fremdbilder der beteiligten Gruppen nachgezeichnet, wenn von ihren sozialen Bindungen und Netzwerken die Rede ist, von ihren verschiedenartigen Auffassungen von individueller Würde und sozialer Ehre, und vor allem geht es immer wieder um den Unterschied zwischen dem weiblichen und dem männlichen Reden über Sexualität und Körperlichkeit. Fast wie in einer zeitgenössischen Sozialreportage erschließt sich uns damit ein Stück gesellschaftliches „Innenleben“ am Beginn der Moderne. Zugleich wird sichtbar gemacht, wie soziale und politische Formen vormoderner Herrschaft in diese Lebenswelten eingreifen, wie Formen traditionellen Rechtsbewußtseins und neue Begriffe moderner Staatlichkeit und Rechtsförmigkeit aufeinanderprallen, wie sich also in den Vergesellschaftungsprozessen des frühen I9. Jahrhunderts grundsätzliche Normen- und Moralkonflikte aufwerfen, die in diesem Prozeß einen exemplarischen Ausdruck finden. „Oberflächlich gesehen, wurden vor Gericht sehr unterschiedliche schicht- und geschlechtsspezifische Moralbegriffe verhandelt. Tatsächlich jedoch spiegelt der Prozeß einen weitreichenden Umbruch im soziokulturellen Gefüge dieser ehemals freien Reichsstadt Hall wider, eine Folge der I 802 vollzogenen Eingliederung des reichsstädtischen Territoriums in das Königreich Württemberg, die das altständische Macht- und Solidargefüge erschütterte. So war es zwar der Umgang mit Prostitution und Sexualität, an dem sich die Auseinandersetzung über bürgerliche Vorstellungen von Moral und Ehre entzündete. Diese Auseinandersetzung war jedoch vor allem Ausdruck eines zähen Machtkampfes zwischen den alten reichsstädtischen Eliten und dem württembergischen Landesherrn um die Durchsetzung eines neuen Herrschaftsreglements. Die Frauen der Haller Unterschicht, deren Selbstversorgungsstrategien jahrelang von der alten Haller Obrigkeit geduldet worden waren, wurden so unversehens zu Opfern eines politischen, sozialen und auch kulturellen Umbruchs." (Kienitz I995: I 8f.)

$\mathrm{Da}$ solche Bilder der Geschichte in dieser wie in anderen Studien so plastisch werden, hängt gewiß auch damit zusammen, daß mikrohistorische Studien gerne auf ethnologische Darstellungsweisen zurückgreifen, wie sie etwa mit dem von Clifford Geertz geprägten Begriff der ,dichten Beschreibung“ skizziert sind. „Dichte Beschreibung“ - darauf wird später ausführlicher zurückzukommen sein meint eine möglichst detaillierte und zugleich komplexe Darstellung einer Situation, deren Abläufe und Beteiligte aus möglichst unterschiedlichen Blickwinkeln und Quellen dargestellt werden; die Diskussion um die - umstrittene - „Rückkehr der Erzählung“ in die Geschichtsschreibung spiegelt diese Anstöße wider. Wenn es auf 
diese Weise gelingt, Vorgänge einerseits minutiös zu rekonstruieren, sie andererseits in ihrer möglichen Mehrdeutigkeit und in der Unterschiedlichkeit ihrer Wahrnehmung durch die sozialen Akteure hermeneutisch zu öffnen, dann werden der Fall, die Szene, die Situation zu einer charakteristischen Momentaufnahme von Kultur und Gesellschaft. Solche detaillierten Momentaufnahmen vermitteln meist mehr Aufschlüsse über die „Praxis der Subjekte“ und über deren gesellschaftliche Rahmenbedingungen als großflächige Beschreibungen.

In diesem Interesse verbinden sich ethnologische und mikrohistorische Studien, weil beide Seiten davon ausgehen, daß es immer verschiedene Deutungshorizonte und auch „Wahrheiten“ sind, auf die wir in Geschichte und Gesellschaft stoßen. Insofern verkörpert jede Geschichtsschreibung immer auch ein Stück kontrollierter Fiktionalität, wenn Lücken interpretierend geschlossen und Schlußfolgerungen nahegelegt werden. Doch im Unterschied zum Roman muß diese Fiktionalität kontrollierbar sein, nämlich deutlich machen, wie diese Verdichtung oder Schlußfolgerung zustande gekommen ist, auf welche Indizien sie sich stützen kann. Dafür bietet die Mikrohistorie mit ihren feingezeichneten Miniaturen, in denen es auf jede Linie und Schraffur ankommt, die besten Voraussetzungen.

\section{Quellen}

Bleibt man in dieser Zeit des Müllers Menoccio oder der Schwäbisch Haller Frauen und damit allein bei schriftlichen Geschichtsquellen, so bietet sich bereits hier ein breites Spektrum unterschiedlicher Textgenres an, das von spärlichen individuellen Zeugnissen wie Briefen und Eingaben über kirchliche und amtliche Berichte, über Chroniken und Gesetzestexte bis zu seriellen Quellen wie Tauf- und Steuerbüchern reicht. Für jede dieser Textarten wie für jeden einzelnen Text meint Quellenkritik die systematische Erschließung in einer doppelten Weise: zum einen die Frage danach, wie der konkrete Text entstanden ist, zum anderen jene nach der Überlieferung und Aufbewahrung dieser Quelle. Wer schrieb, für wen, zu welchem Anlaß und Zweck, in welchem weiteren historischen Zusammenhang, in welcher "Sprache“, und weshalb und wo wurde der Text aufgehoben? Diese Fragen nach dem Entstehungs- und Überlieferungszusammenhang von Quellen verlangen von der Quellenkritik die Anwendung systematischer Methoden der Textbearbeitung und der Quelleninterpretation - vielleicht mehr noch als in der „Makrogeschichte", weil dem einzelnen Quellenstück hier größeres Gewicht zukommt und weil es sich stärker gegen die Einordnung in Modelle und Interpretationsraster ,wehrt“ als ein großes Quellenkorpus, das allein schon durch die zu bewältigende Masse seiner Informationen 
eher eine auch argumentativ ,,passende“ Selektivität erlaubt bzw. erzwingt.

Man kann darüber streiten, ob es sinnvoll ist, Teile der älteren wie jüngeren volkskundlichen Dorfforschungen ebenfalls unter die Überschrift „Mikrohistorie“ zu stellen. Es waren und sind jedenfalls auch Versuche, historische oder von der Geschichte besonders stark imprägnierte Alltagswelten und -kulturen in eng begrenzten Ausschnitten intensiv auszuleuchten und dabei die vorhandenen Quellen möglichst „total“ zu erfassen, um ihnen historische Erfahrungen, Weltanschauungen, Biographien zu entlocken. ${ }^{64}$ Auch dieses Ausschnittformat von Lokalgesellschaften und Dörfern hat sich jedoch für manche Fragestellung und manchen Quellenbestand noch als zu groß erwiesen. Vor allem dann, wenn lange historische Entwicklungslinien eingefangen werden sollen, wird das Feld daher noch weiter verkleinert auf den Ausschnitt einer einzelnen Familie oder eines Hauses. Es sind oft methodische Forschungsexperimente, die dabei unternommen werden müssen, aber ihr Reiz und ihr möglicher Erkenntnisgewinn lassen sich schlüssig begründen: „Ein Haus ist in noch stärkerem Maße ,Einzelnes` als eine Region, ein bestimmtes Dorf, eine Stadt, markiert einen noch kleineren Ausschnitt aus der gesellschaftlichen und geschichtlichen Wirklichkeit als diese. (...) In erster Linie geht die ,Geschichte eines Hauses' der Frage nach, inwieweit sich die ,totale' Erfassung eines extrem kleinen Mikroraums als methodischer Zugang zur Lebenswelt der ,kleinen Leute', die kaum eigene schriftliche Zeugnisse hinterlassen haben, nutzen läßt." (Hochstrasser I993: I0)

Nicht zuletzt über diese volkskundliche Dorf- und Gemeindeforschung ist immer stärker auch die Gegenwartserforschung an die Mikrogeschichte herangerückt worden. Damit sind vielfach andere, zum Teil nichtschriftliche Quellen in die Auswertung einbezogen, in denen nun tatsächlich ,die Subjekte“ selbst sprechen. Erzählungen etwa und lebensgeschichtliche Interviews tauchen (nicht nur) Dörfer und Vergangenheiten scheinbar automatisch und zuverlässig in den warmen Schein jener authentisch verbürgten Erfahrung, nach der wir suchen. Zweifellos sind solche mündlichen Quellen entscheidende Zeugnisse individueller wie kollektiver Erinnerung und damit einer spezifischen geschichtlichen Logik, ${ }^{65}$ allerdings werfen sie methodisch nicht weniger Probleme der Analyse und Interpretation auf als schriftliche Texte. Die volkskundliche Erzählforschung, die sich seit langem mit Stoffen und Fragen mündlicher Tradierung beschäftigt, hat den Trend zur Oral History etwa in der Geschichtswissenschaft daher nicht nur mit Begeisterung verfolgt, da dort manche der theoretischen und methodologischen Fußangeln im Erzählfeld zunächst übersehen worden sind: die Wirkung von Gattungs- und Formtradi- 
tionen etwa oder Fragen der Phraseologie- und Toposforschung. Denn erzählte Erinnerung folgt vielfältigen kulturellen Regeln der Stoff- und Formgestaltung. Insofern sollten solche Muster des Redens und Erzählens ,nicht allein als augenblickliche Gestaltung gesehen werden, was sie stets auch sind, sondern in ihren historischen und kulturellen Voraussetzungen als Formen des individuellen und kollektiven Bewußtseins analysiert werden." (Lehmann I993: 430)

Bei einer weiteren Quellenart stellen sich ganz ähnliche Probleme der Lesart und Entzifferung, nämlich bei Fotografien. Sie werden als mikro- und alltagsgeschichtliche Quelle für die Zeit nach der Mitte des I9. Jahrhunderts in den letzten Jahren vermehrt genutzt, und auch sie bieten zunächst eine Oberfläche an, die scheinbar eindeutige Dokumentationen vergangenen Lebens liefert. Beim zweiten Blick jedoch stellt ihr „Lesen“ uns vor entsprechende Probleme der Deutung einer fremden Handschrift wie ein Text aus dem I7. Jahrhundert, und andere, medienspezifische Fragen gesellen sich noch dazu. „Die Fotografie erfüllt die Kriterien, die einer Quelle zukommen, denn sie ist - wie ein Brief oder eine Akte - medialer Träger von Informationen. Die Fotografie hat, ganz gleich, ob es sich um ein Amateurfoto, ein Pressefoto oder eine künstlerische Fotografie handelt, immer auch dokumentarischen Wert. Sie kann als biographisches, soziales, kulturgeschichtliches und als historisches Dokument gelesen werden. (...) Gleichwohl muß der Historiker die Informationen, die sich dem Medium in einer bestimmten Form eingeschrieben haben, ... erst erschließen und interpretieren, damit sie zu einer historischen Aussage werden können." (Berg I994: I 87)

Es gilt also einerseits, die Entstehung des Fotos und seine zeitgenössischen Gebrauchsweisen zu rekonstruieren, über deren aktuellen Zustand der Kultursoziologe Pierre Bourdieu notiert hat: „Obgleich sich das Spektrum des Photographierbaren ständig erweitert, ist die photographische Praxis deswegen nicht freier, da man nur das photographieren darf, was man photographieren muß, und weil es Bilder gibt, die man ,unbedingt aufnehmen' muß, so wie es Naturschönheiten und Monumente gibt, die man ,mitnehmen' muß. Traditionellen Funktionen unterworfen, bleibt die Praxis deshalb auch in der Wahl der Objekte, der Augenblicke und sogar in ihrer Intention traditionell." (Bourdieu I983: 49) Andererseits ist unser eigener Blick auf dieses Dokument fremder Praxis zu problematisieren, der immer in Gefahr steht, das hineinzusehen, was er sucht, die Fotografie also als buchstäbliche Illustration seiner These zu nehmen. Trotz der vielseitigen Erfahrungen und Methoden vor allem der Kunstgeschichte im Umgang mit Bildern hinkt hier die kulturgeschichtliche Bildforschung der textlichen noch weit hinterher. ${ }^{66}$ 
„Research up“ in der Geschichte?

Eingangs habe ich darauf hingewiesen, daß der Begriff Mikrohistorie zwar zunächst forschungspraktisch für eine Geschichte der „kleinen Dinge“ und der „,kleinen Leute“ stand. Man wollte damit mehr erfahren über jene Menschen und Alltage, die vorher eher im Schatten der Geschichtsschreibung gestanden hatten. Doch erschöpft sich darin der Sinn mikrohistorischer Forschung längst nicht mehr, vor allem nachdem deutlich geworden ist, welche anderen Perspektiven dieser Zugang auch auf die Abläufe einer ,großen Geschichte“ zu vermitteln vermag. So hat der Historiker Georges Duby einen Tag der Schlacht von Bouvines im Jahre I2 I4 minutiös rekonstruiert, um die militärischen Abläufe, die geographischen und meteorologischen Bedingungen, die durch Quellen verfügbaren Details sichtbar zu machen, die letztlich über Sieg oder Niederlage entschieden und die damit „Weltgeschichte“ machten: England verlor in der Folge dieser Schlacht zwischen den siegreichen französischen Truppen Philipps II. August und denen von Kaiser Otto IV. den größten Teil seines europäischen Festlandbesitzes, Otto den deutschen Thron an die Staufer (Duby 1988). Duby demonstriert hier an einem kleinen und zugleich großen Beispiel, daß und wie Geschichte nicht nur von ihren Ergebnissen her zu lesen, sondern in ihren konkreten Bedingungen und Abläufen zu verfolgen ist, wenn ihr Gang nicht nur als Gesetzmäßigkeit ex post gedeutet werden soll. Er verweist damit - anknüpfend auch an die französische Tradition einer Geschichte der Mentalitäten - auf weitere Möglichkeiten einer „totalen“ Form der Geschichtsschreibung bzw. ihrer Quellenauswertung (Schöttler I989).

Mikrogeschichte - in diesem Sinne angewandt - vermag also auch einer „Geschichte der Macht“ wesentliche neue Erkenntnisse hinzuzufügen, indem sie die vermeintlich großen strategischen Entscheidungen gegebenenfalls in kleine Schritte und Zufälle auflöst, indem sie große Figuren von ihrem Podest holt, indem sie Geschichte ,,vermenschlicht" in begrenzte Erfahrungsdimensionen, individuelle Handlungsweisen und schließlich subjektive Gefühle. Ihr Blick, der sich lange Zeit überwiegend „nach unten“ gerichtet hat, richtet sich inzwischen ebenso "nach oben“. Auch hier kündigt sich jenes research up wie in der ethnologischen Feldforschung an, und auch hier trägt es in Umkehrung dieser Metapher in Wirklichkeit dazu bei, Geschichte wieder ,auf den Boden“ ihrer Bedingungen wie unserer Betrachtungen zurückzuholen. 


\section{Materielle Kultur: Die Sprache der Dinge}

Wenn wir von Kultur sprechen, haben wir meist die Beziehungen und Kommunikationsformen zwischen Menschen vor Augen und vergessen dabei leicht, daß zu dieser Kultur auch jene Dinge gehören, in denen sich unsere sozialen Beziehungen materialisieren und symbolisieren: als Produkte von Arbeit, als Gegenstände des täglichen Gebrauchs, als bebaute Umwelten, als Objekte mit Zeichen- und Erinnerungsfunktion. „Die Dinge“ in diesem weiten Sinne sind Kristallisationen unserer kulturellen Praxis, weil sich in ihnen Denkweisen, Wertehorizonte und Nutzungsformen „,verewigen“, die ansonsten in Gespräch und Gebrauch so rasch wieder verschwunden sind, wie wir sie zitieren und anwenden. Die ästhetische Gestaltung eines Schmuckstücks, die architektonische Gliederung einer Hausfassade, der von Händen abgenutzte Stiel eines Hammers - das sind beabsichtigte wie ungeplante Spuren kultureller Tätigkeit, die sich uns entäußern, indem sie auf den Gegenstand übergehen. Dieser nimmt damit unseren Blick und unsere Handschrift in sich auf, gleichsam Bestandteile unserer Identität, die sich so auch außerhalb unserer selbst widerspiegeln. Wenn wir also von der "Sprache der Dinge“ reden, dann schreiben wir ihnen kein eigenes Leben, keine eigene Artikulationsfähigkeit zu, sondern meinen diese eingeschriebene Träger- und Bedeutungsfunktion, die Gegenstände durch unsere Nutzung und Gestaltung erhalten.

\section{Zeugnis und Mythos}

Diese besondere Eigenschaft der Dinge, Nutzungen und Bedeutungen gleichsam in ihrer physikalisch-materiellen Beschaffenheit zu speichern, haben sich schon die frühe Volkskunde und Völkerkunde in eigener Weise zunutze gemacht. Die Dinge, vor allem jene, die von den Menschen einem besonderen symbolischen oder sakralen Zweck gewidmet waren, wurden als sachhafte Zeugnisse einer vergangenen Weltsicht betrachtet, die sich in eigenen Stimmen und Texten ansonsten kaum überliefert hatte. Afrikanische Amulette oder germanische Runen schienen Schlüsselsymbole vergangener kultureller wie kultischer Praktiken zu verkörpern, deren mythologischer Horizont sich anhand von Gestaltungsweisen und Gebrauchsspuren rekonstruieren lassen mußte. Insbesondere die Konzeption der frühen Völkerkundemuseen zeugt von dieser Auffassung und einer damit verbundenen Sammelpraxis, die das Sakrale und Kultische als Kristallisationskern wie als Schlüssel zum Verständnis des Denkens 